\title{
Mutation screening of the FKBPG gene and its association study with spermatogenic impairment in idiopathic infertile men
}

\author{
Wei Zhang, Sizhong Zhang, Cuiying Xiao, Yuan Yang and A Zhoucun \\ Divison of Human Morbid Genomics, State Key Laboratory of Biotherapy, Department of Medical Genetics, West \\ China Hospital, Sichuan University, Renmin Nanlu, Section 3 \#17, Chengdu 610041, People's Republic of China \\ Correspondence should be addressed to S Zhang; Email: szzhang@mcwcums.com
}

\begin{abstract}
Fkbp6 has been proved to be a new component of synaptonemal complexes and be involved in homologous chromosomes pairing and male infertility in mice. To explore the possible association between variations in the FKBP6 gene and impaired spermatogenesis in human, mutation screening of all the eight exons and the intron/exon boundaries of the gene was performed in 323 patients with azoospermia or severe oligozoospermia and 205 fertile controls by denatured HPLC and DNA sequencing. As a result, four novel and one known single nucleotide transitions were identified, including c.58-2A $>G, c .111 C>T, c .156 G>T$, c.594G $>A$, and c.216C $>A$ (rs3750075). The frequencies of genotype CA, allele A of c.216C $>A$ and haplotype 'GAG' consisting of c.156G $>$ T, c.216C $>A$, and c.594G $>A$ were significantly lower in infertile patients than those in controls. These findings suggest that the FKBP6 gene may play a role in modifying the susceptibility to idiopathic spermatogenic impairment in human and propose that the allele $A$ of $c .216 C>A$ seems to be a protective factor for the development of male infertility.

Reproduction (2007) 133 511-516
\end{abstract}

\section{Introduction}

As a major health problem, infertility occurs in approximately $15 \%$ of couples worldwide and about half of it is due to male factors (de Kretser 1997, Matzuk \& Lamb 2002). It is generally accepted that genetic defects play an important role in etiology of idiopathic male infertility, and in genetically modified or mutant animal models, hundreds of candidate genes related to spermatogenic impairment have been identified (de Rooij \& de Boer 2003, Scherthan 2003, Vogt 2004, O'Bryan \& de Krester 2006). However, to date very few have been confirmed in human (de Kretser 1997, Matzuk \& Lamb 2002). Therefore, it is reasonable to postulate that at least some of these genes may also play a role in impaired spermatogenesis in human.

Synaptonemal complexes (SCs) are elaborate meiosisspecific supramolecular proteinaceous structures involving in pairing and recombination of homologous chromosomes during meiosis (Heyting 1996, Zickler \& Kleckner 1999). Genetic variations in SC genes may contribute to human male infertility (Page \& Hawley 2004). For example, Miyamoto et al. (2003) found a heterozygous deletion of one base of synaptonemal complex protein 3 (SCP3) gene in two patients with azoospermia, which resulted in a premature stop codon and truncation of SCP3, and proposed that the mutation was associated with non-obstructive azoospermia in human. Other two research groups also reported some abnormalities of SCP, probably in SCP1, in patients with azoospermia (Judis et al. 2004, Sun et al. 2004). In addition, polymorphisms of certain meiosis-related genes were reported to be associated with spermatogenic impairment in human (Sato et al. 2006, Zhoucun et al. 2006).

Recently, Fkbp6 has been proved to be a new component of SCs (Crackower et al. 2003). In Fkbp6 gene knockout mice, the male mice are infertile with azoospermia and absence of normal pachytene spermatocyte, but female mice show no apparent abnormalities in all aspects, including fertility (Crackower et al. 2003). In human, the FKBP6 gene is located on chromosome $7 q 11.23$ and expresses in various tissues with the highest expression in testis. The gene product, FK-506-binding protein 6 (FKBP6), belongs to the immunophilins FKBP family. It contains a three-unit tetratricopeptide repeat motif and has peptidyl-prolyl cis-trans isomerase activity (Meng et al. 1998).

With the essential role of the Fkbp6 gene and SCPs in male reproduction in mind, we postulated that variations in human FKBP6 gene might be associated with impaired spermatogenesis, and performed a mutation 
screening of the FKBP6 gene in 323 infertile patients with azoospermia or severe oligozoospermia and compared the results with those of 205 controls.

\section{Materials and Methods}

\section{Patients and controls}

All patients and controls participating in this study were genetically unrelated and recruited from West China Hospital, Sichuan University. The total 323 patients, aged from 23 to 38 years, included 218 infertile men with idiopathic azoospermia and 105 infertile men with severe oligozoospermia (sperm concentration $<5 \times$ $10^{6} \mathrm{sperm} / \mathrm{ml}$ ). All of them underwent at least twice semen analyses according to the World Health Organization Guidelines (1999) and their chromosomal abnormalities, including microdeletions of azoospermia factor (AZF) region on $\mathrm{Y}$ chromosome were excluded by cytogenetic and corresponding molecular examinations (Simonim et al. 1999). The control group comprised 205 fertile men, aged from 26 to 45 years, who had fathered at least one child without any assisting reproductive techniques. This study was approved by the Institutional Ethic Review Board of West China Hospital, Sichuan University and informed consents were obtained from all participants.

\section{PCR amplification}

Genomic DNA was extracted from peripheral blood lymphocytes using standard phenol-chloroform procedures. To amplify all the eight exons, the intron/exon boundaries and the non-coding region upstream of exon 1 , eight pairs of primers were designed according to the mRNA sequence (GenBank Accession no. NM_003602) and human genomic sequence using software Primer
Premier 5.0 (Table 1). PCRs were carried out in a total volume of $30 \mu \mathrm{l}$ containing $0.1 \mu \mathrm{g}$ genomic DNA, $7 \mathrm{pmol}$ each primer, 6 pmol dNTP, 1.5 units Taq polymerase (TaKaRa, Shiga, Japan), and standard PCR buffer. After predenaturation at $94{ }^{\circ} \mathrm{C}$ for 5 min, 35 amplification cycles were performed with a temperature profile consisting of denaturation at $94{ }^{\circ} \mathrm{C}$ for $30 \mathrm{~s}$, annealing at a temperature between 52 and $62{ }^{\circ} \mathrm{C}$ for $30 \mathrm{~s}$, and extension at $72{ }^{\circ} \mathrm{C}$ for $1 \mathrm{~min}$, followed by an extra final extension at $72{ }^{\circ} \mathrm{C}$ for $5 \mathrm{~min}$. The annealing temperatures for amplifying each DNA fragment were shown in Table 1.

\section{Denatured HPLC (DHPLC) analysis and DNA sequencing}

The automated WAVE 3500HT Nucleic Acid Fragment Analysis System (Transgenomic, Inc., Omaha, NE, USA) was applied to screen variations in the FKBP6 gene. The WAVEMAKER4.1 (Transgenomic) software was used to determine the optimal melting temperatures for the amplified fragments (Table 1). Prior to DHPLC analysis, PCR products were denatured at $94{ }^{\circ} \mathrm{C}$ for $5 \mathrm{~min}$ and cooled at room temperature over $45 \mathrm{~min}$. Then, $5 \mu \mathrm{l}$ products were injected into a high-throughput DNASep column (Transgenomic) and eluted with a linear acetonitrile gradient of $2 \%$ per minute at a flow rate of $0.9 \mathrm{ml} / \mathrm{min}$. The elution profiles of heterozygous fragments were represented as multiple peaks and/or aberrant shaped peak. In contrast, homozygous fragments showed a single peak. After DHPLC analysis, heterozygous fragments were reamplified and purified using QIAquick PCR purification Kit (Qiagen) and then underwent direct sequencing in both directions on ABI377 DNA Sequencer (Applied Biosystems, Foster City, CA, USA). Several PCR products were cloned into pGEM-T Easy Vector System (Promega) and sequenced (Fig. 1).

Table 1 The PCR amplified regions, primer sequences, annealing temperatures, product size, and the melting temperature of denaturing HPLC (DHPLC) for mutation screening.

\begin{tabular}{|c|c|c|c|c|}
\hline Amplified region & Primer sequence $\left(5^{\prime}-3^{\prime}\right)$ & Annealing temperature $\left({ }^{\circ} \mathrm{C}\right)$ & Product size $(b p)$ & Melting temperature $\left({ }^{\circ} \mathrm{C}\right)$ \\
\hline \multirow[t]{2}{*}{ Exon 1} & F: AATGCCGCCGTCGGTAGGGG & 62 & 200 & 63.1 \\
\hline & R: ACGTCTGGGCCСТCACCTGG & & & \\
\hline \multirow[t]{2}{*}{ Exon 2} & F: CGTAGACGCTGAGGGGTGGC & 60 & 240 & 62.3 \\
\hline & R: TCTTCCCAACAGAGTGCGATGA & & & \\
\hline \multirow[t]{2}{*}{ Exon 3} & F: TCCTGTACCTCGTGGTGTCGG & 55 & 281 & 58.1 \\
\hline & R: GGTGCCTCATCTCGСТССТАAAC & & & \\
\hline \multirow[t]{2}{*}{ Exon 4} & F: GGGGAAGAAACTGATAGCATTA & 55 & 329 & 58.7 \\
\hline & R: ACACCAACCСАTTTGTCTCTCTA & & & \\
\hline \multirow[t]{2}{*}{ Exon 5} & F: CACTTCCATTGTGGCATTA & 52 & 200 & 58.3 \\
\hline & R: TTCAAAGCATTCTCACСCT & & & \\
\hline \multirow[t]{2}{*}{ Exon 6} & F: TAGTTTTTGCGTGTGGATTCTTTA & 55 & 320 & 59.9 \\
\hline & R: CCTTTTCCTGGGTGTTTATTC & & & \\
\hline \multirow[t]{2}{*}{ Exon 7} & F: CACCGTGCCCAGCCAAATGATA & 56 & 296 & 59.6 \\
\hline & R: GGGGGCGGCCTCCTAACA & & & \\
\hline \multirow[t]{2}{*}{ Exon 8} & F: TCAССТССТССАААСАСАGАСС & 55 & 235 & 59.8 \\
\hline & R: TGCAGACAGCCACCATTCACT & & & \\
\hline
\end{tabular}

All PCRs were carried out in $1.5 \mathrm{mM} \mathrm{MgCl}_{2}$ with exception of exon 2 in $2 \mathrm{mM} \mathrm{MgCl}_{2}$. 

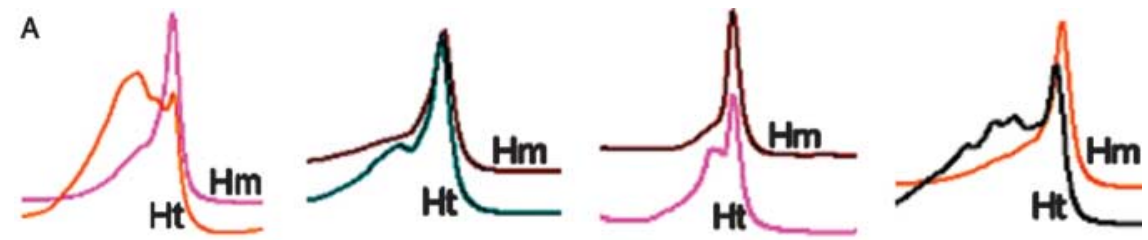

B
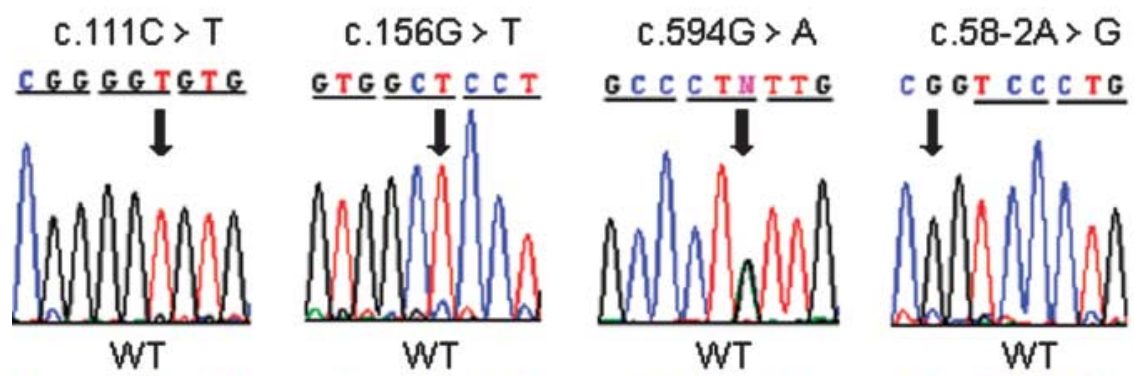

WT
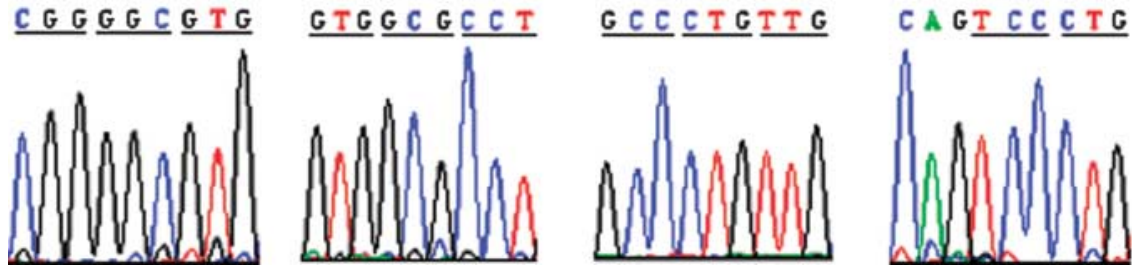

Figure 1 Detection and confirmation of the four novel single nucleotide transitions in the FKBP6 gene by DHPLC and DNA sequencing. (A) DHPLC elution profiles: homozygous fragments with a single peak and heterozygous fragments with an aberrant peak. (B) DNA sequences of the four variations: mutant sequences (upper panel) and wide-type sequences (lower panel). Arrows indicate the positions of the variations and codons are underlined by dark horizontal lines. The nomenclature of variations follows to the recommendations in website http://www.hgvs.org/mutnomen/.

\section{Genotyping}

Genotyping for the single nucleotide polymorphisms (SNPs) identified in this study was carried out by PCRrestriction fragment length polymorphism (PCR-RFLP) analysis with corresponding restriction enzymes (Hhal, Taql, and AlwNI; NEB, Beverly, MA, USA). After restriction enzyme digestion, the products were electrophoresed on a $2 \%$ agarose gel and observed with Gel Doc1000 system (Bio-Rad).

\section{Statistical analysis}

Hardy-Weinberg equilibrium (HWE) program was used to test the deviation from HWE for alleles. Differences in genotypic and allelic frequencies of SNPs between infertile patients and controls were assessed by $\chi^{\text {? test }}$ using SPSS12.0 software. Pairwise linkage disequilibrium (LD) coefficients $\left(D^{\prime}\right)$ were calculated using $2 \mathrm{LD}$ program (http://www.mrc-epid.cam.ac.uk/Personal/jinghuazhao/software; Zhao 2004). Program PHASE 2.1.1 using Bayesian algorithm (Stephens et al. 2001, Stephens \& Donnelly 2003) was utilized to reconstruct haplotypes from genotype data and to evaluate their possible association with impaired spermatogenesis. The frequency differences of individual haplotypes between patients and controls were also assessed by $\chi^{2}$-test using SPSS12.0 software.

\section{Results}

By screening all the eight exons, the intron/exon boundaries and the $5^{\prime}$ UTR of the FKBP6 gene in 323 infertile men with oligo-/azoospermia and 205 controls, five single nucleotide transitions were detected and then confirmed by DNA sequencing. The nomenclature of these sequence variations followed the recommendations in the website http://www.hgvsorg/mutnomen. Among them, c.216C $>$ A was the only non-synonymous variation resulting in substitution of Phe by Leu at codon 72 (p.Phe72Leu) and had been listed in the NCBI dbSNP database (ID: rs3750075). The other four were novel, including three synonymous variations (c.111C $>\mathrm{T}$, c.156G $>$ T, and c.594G $>A$ ) and one variation in the consensus splice site (c.58-2A $>\mathrm{G}$ ).

Both c.111C $>$ T and c.58-2A $>$ G were observed only in one patient each. By PCR-RFLP analysis, we discovered that the frequencies of all the three minor alleles of c.156G > T, c.216C > A, and c.594G > A were over $1 \%$ in both infertile patients and controls, so they were SNPs (Table 2). The genotype distributions of the three SNPs were in HWE in both groups (data not shown). As shown in Table 2, the frequencies of allele A and genotype CA of c.216C > A were significantly lower in patients with oligo-/azoospermia than those in controls $(P<0.001)$ that was consistent with our previous study on 177 patients with azoospermia 
Table 2 Distributions of genotypic and allelic frequencies of three single nucleotide polymorphisms in the FKBP6 gene of infertile patients and controls.

\begin{tabular}{lllll}
\hline & & \multicolumn{2}{c}{ Genotype and allele frequency } \\
\cline { 3 - 5 } SNP & Genotype allele & Patients $(n=323)$ & Controls $(n=205)$ & \multicolumn{1}{c}{$\boldsymbol{P}$ value } \\
\hline c.156G $>$ T & GG/GT/TT & $294 / 29 / 0$ & $189 / 16 / 0$ & $\mathrm{NS}$ \\
& G/T & $617(95.5) / 29(4.5)$ & $394(96.1) / 16(3.9)$ & \\
c.216C $>$ A & CC/CA/AA & $312 / 11 / 0$ & $183 / 22 / 0$ & 0.0006 \\
c.594G $>$ A & C/A & $635(98.3) / 11(1.7)$ & $388(94.6) / 22(5.4)$ & \\
& GG/GA/AA & $276 / 46 / 1$ & $169 / 36 / 0$ & $\mathrm{NS}$ \\
& G/A & $598(92.6) / 48(7.4)$ & $374(91.2) / 36(8.8)$ & $\mathrm{NS}$ \\
\hline
\end{tabular}

Data of genotype and allele frequency are presented as ' $n$ ' and ' $n(\%)$ ' respectively. NS, not significant.

(Zhang et al. 2005). Moreover, the allele A of c.216C >A showed a protective effect against male infertility in human (by allele A: $\mathrm{OR}=0.306,95 \% \mathrm{Cl}$ : $0.147-0.737$; by genotype CA: $\mathrm{OR}=0.293,95 \% \mathrm{Cl}$ : $0.139-0.619)$. No significant differences in genotypic or allelic frequencies of both c. $156 \mathrm{G}>\mathrm{T}$ and c.594G $>$ A between patients and controls were observed.

The coefficients $\left(D^{\prime}\right)$ of pairwise LD in the control group presented in Table 3 indicated that there was no complete LD among the three SNPs, c.156G $>$ T, c. 216C $>$ A, and c.594G $>$ A. In total, seven haplotypes consisting of these SNPs were observed and their frequencies in both patients and controls were estimated by PHASE2.1.1 program. As shown in Table 4, four haplotypes with frequency of more than $1 \%$ accounted for over $95 \%$ of the total haplotypes. The PHASE casecontrol global test revealed remarkable difference of haplotype distribution between infertile patients and controls (global $P=0.01$ ), which suggested a significant association between the haplotypes and human male infertility. Comparison of individual haplotypes between the two groups showed that the frequency of haplotype 'GAG' consisting of c.156G > T, c.216C $>A$, and c.594G >A was significantly decreased in infertile patients compared with controls (1.66 vs $4.86 \%$, $P=0.0029$; Table 4).

\section{Discussion}

In order to explore the genetic causes of male infertility in human, mutation screening in patients with spermatogenic impairment may be the most effective approach at present time. Therefore, we screened the FKBP6 gene and performed association study of the gene with male infertility. As the result, five single nucleotide transitions were detected, including three SNPs and two rare variations. Except the c.216C $>A$ (rs3750075), other four transitions were newly discovered. Since the distributions of two novel SNPs, c.156G $>$ T and c.594G $>$ A, were similar between patients and controls $(P>0.05)$, they might be normal polymorphisms. The c.111C $>\mathrm{T}$ was a synonymous variation with very scarce frequency, and it might not be a disease-associated variation. Although c.58-2A $>\mathrm{G}$ changed the consensus splice site, its effect on spermatogenesis needed further research, because it was found in only one azoospermic man.

The frequencies of allele $A$ and genotype $C A$ of c.216C $>A$ were significantly lower in patients with oligo-/azoospermia than those in controls $(P<0.001)$. In addition, the odds ratios (ORs) suggested that the carriers with allele $\mathrm{A}$ had lower risk compared with those with allele C. Therefore, the allele A seemed to have a protective effect against development of male infertility. Besides, we further used the web-based program ESEfinder (Exonic Splicing Enhancer (ESE); http://rulai.cshl.edu/tools/ESE/; Cartegni et al. 2003) to predict the effects of c. $216 \mathrm{C}>\mathrm{A}$ on activity of premRNA splicing. It was shown that the substitution of C by A of SNP c.216C $>$ A created a new exonic splicing enhancer consensus sequence (GACCCTTA), and that the consensus sequence could be recognized and bound by the splicing factor SC35, which was an important member of serine/arginine-rich protein family playing a key role in pre-mRNA splicing (Cartegni et al. 2003, Sanford et al. 2005). Therefore, the allele A of c.216C $>$ A may enhance the activity of pre-mRNA splicing and further increase the expression of the FKBP6 gene compared with allele C. So the SNP c.216C>A may be a functional polymorphism in the FKBP6 gene and it is worthy of attention in the functional studies of the gene.

Since no complete $\operatorname{LD}\left(D^{\prime}=1\right)$ was observed among the three SNPs, c.156G > T, c.216C >A, and c.594G >A, we performed further haplotype analysis of them in the patients and controls and found a significant association

Table $3 D^{\prime}$ values of pairwise linkage disequilibrium among the three single nucleotide polymorphisms (SNPs) in the FKBP6 gene.

\begin{tabular}{lcc}
\hline SNP & c.156G $>$ T & c.216C $>$ A \\
\hline c.216C $>$ A & 0.701 & \\
C.594G $>$ A & 0.884 & 0.245 \\
\hline
\end{tabular}

Linkage disequilibrium was calculated for the control group. 
Table 4 Frequencies of the haplotype consisting of three single nucleotide polymorphisms (SNPs) in the FKBP6 gene estimated using PHASE2.1.1 program and comparisons between infertile patients and controls.

\begin{tabular}{lccc}
\hline Haplotypes & Control $\boldsymbol{F}$ & Patient $\boldsymbol{F}$ & $\boldsymbol{P}$ value \\
\hline GCG & 0.826736 & 0.868998 & NS \\
GCA & 0.081137 & 0.069454 & NS \\
GAG & 0.048592 & 0.016656 & 0.0029 \\
GAA & 0.004510 & 0.000000 & NS \\
TCG & 0.036311 & 0.039671 & NS \\
TCA & 0.002157 & 0.004849 & NS \\
TAG & 0.000556 & 0.000372 & NS \\
\hline
\end{tabular}

Three SNPs constituting haplotypes: c.156G > T, c.216C $>$ A, and c.594G $>$ A. $P=0.01$ for PHASE case-control global test. Control $F$, haplotype frequency estimated from controls; patient $F$, haplotype frequency estimated from patients. NS, not significant.

of haplotypes with male infertility using PHASE casecontrol global test (global $P=0.01$ ). Comparison of individual haplotypes showed that the frequency of haplotype 'GAG' consisting of the three SNPs was significantly lower in patients than that in controls (1.66 vs $4.86 \%, P=0.0029$ ). All of these suggested that the FKBP6 gene might modify the human susceptibility to spermatogenic impairment, and also supported the protective effect of allele A of SNP c.216C $>$ A. Alternatively, it could not be excluded that the c.216C $>$ A might be in LD with another functional locus nearby.

Meanwhile, a recent study reported that the haploinsufficiency for the FKBP6 gene did not cause azoospermia (Metcalfe et al. 2005). Another research on 51 men with non-obstructive azoospermia failed to find mutations in the FKBP6 gene including the c.216C $>\mathrm{A}$ and proposed that the gene mutations were not a common cause of azoospermia (Westerveld et al. 2005). Our study was based on a larger sample size with 528 subjects. Although no apparent mutations leading to oligo-/azoospermia were detected, the results of the association studies strongly suggested a role of the FKBP6 gene in modifying the susceptibility to idiopathic spermatogenic impairment in human and proposed that the allele A of c.216C $>$ A seemed to be a protective factor for development of male infertility. Therefore, further more in-depth researches with larger samples in more populations and the functional studies of some variations in the FKBP6 gene are desirable.

\section{Acknowledgements}

This work was supported by the National Natural Science Foundation of China (Grant no. 30470960), National Basic Research Program of China (Grant No.2004CB518805), and China Medical Board Foundation of New York. The authors declare that there is no conflict of interest that would prejudice the impartiality of this scientific work.

\section{References}

Cartegni L, Wang J, Zhu Z, Zhang MQ \& Krainer AR 2003 ESEfinder: a web resource to identify exonic splicing enhancers. Nucleic Acids Research 31 3568-3571.

Crackower MA, Kolas NK, Noguchi J, Sarao R, Kikuchi K, Kaneko H, Kobayashi E, Kawai Y, Kozieradzki I, Landers R et al. 2003 Essential role of Fkbp6 in male fertility and homologous chromosome pairing in meiosis. Science 300 1291-1295.

de Kretser DM 1997 Male infertility. Lancet 349 787-790.

De Rooij DG \& de Boer P 2003 Specific arrests of spermatogenesis in genetically modified and mutant mice. Cytogenetic and Genome Research 103 267-276.

Heyting C 1996 Synaptonemal complex: structure and function. Current Opinion in Cell Biology 8 389-396.

Judis L, Chan ER, Schwartz S, Seftel A \& Hassold T 2004 Meiosis I arrest and azoozpermia in an infertile male explained by failure of formation of a component of the synaptonemal complex. Fertility and Sterility 81 205-209.

Matzuk MM \& Lamb DJ 2002 Genetic dissection of mammalian fertility pathway. Nature Medicine 8 S41-S49.

Meng X, Lu XJ, Colleen AM \& Keating MT 1998 A novel human gene FKBP6 is deleted in William Syndrome. Genomics $\mathbf{5 2}$ $130-137$.

Metcalfe K, Simeonov E, Beckett W, Donnai D \& Tassabehju M 2005 Autosomal dominate inheritance of Williams-Beuren syndrome in a father and son with haploinsufficiency for FKBP6. Clinical Dysmorphology 14 61-65.

Miyamoto T, Hasuike S, Yogev L, Maduro MR, Ishikawa M, Westphal H \& Lamb DJ 2003 Azoospermia in patients heterozygous for a mutation in SCP3. Lancet 362 1714-1719.

O'Bryan MK \& de Kretser D 2006 Mouse models for genes involved in impaired spermatogenesis. International Journal of Andrology 29 76-89.

Page SL \& Hawley RS 2004 The genetics and molecular biology of the synaptonemal complex. Annual Review of Cell and Developmental Biology 20 525-558.

Sanford JR, Ellis J \& Caceres JF 2005 Multiple roles of arginine/serinerich splicing factors in RNA processing. Biochemical Society Transactions 33 443-446.

Sato $H$, Miyamoto T, Yogev L, Namiki M, Koh E, Hayashi $H$, Sakaki Y, Ishikawa M, Lamb DJ, Matsumoto N et al. 2006 Polymorphic alleles of the human MEI1 gene are associated with human azoospermia by meiotic arrest. Journal of Human Genetics 51 533-540.

Scherthan H 2003 Knockout mice provide novel insights into meiotic chromosome and telomere dynamics. Cytogenetic and Genome Research 103 235-244.

Simonim M, Bakker E, Eurlings MCM, Matthijs G, Moro E, Muellers CR \& Vogt PH 1999 Laboratory guidelines for molecular diagnosis of Y-chromosomal microdeletions. International Journal of Andrology 22 292-299.

Stephens M \& Donnelly P 2003 A comparison of Bayesian methods for haplotype reconstruction from population genotype data. American Journal of Human Genetics 73 1162-1169.

Stephens M, Smith N \& Donnelly P 2001 A new statistical method for haplotype reconstruction from population data. American Journal of Human Genetics 68 978-989.

Sun F, Kozak G, Scott S, Trpkov K, Ko E, Mikhaail-Philips M, Bestor TH, Moens P \& Martin RH 2004 Meiotic defects in a man with nonobstructive azoospermia: case report. Human Reproduction 19 1770-1773.

Vogt PH 2004 Molecular genetics of human male infertility: from genes to new therapeutic perspective. Current Pharmaceutical Design 10 471-500.

Westerveld GH, Repping S, Lombardi MP \& van der Veen F 2005 Mutations in the choromosome pairing gene FKBP6 are not a common cause of non-obstructive azoospermia. Molecular Human Reproduction 11 673-175. 
Zhang W, Zhang SZ, Xiao CY, Yang Y, Ma YX, Cheng L, A ZC, He GP \& Shi JJ 2005 Possible association between 278C/A single nucleotide polymorphism of FKBP6 and idiopathic azoospermia. Chinese Journal of Medical Genetics 22 10-13.

Zhao JH 2004 2LD, GENECOUNTING and HAP: Computer programs for linkage disequilibrium analysis. Bioinformatics 20 1325-1326.

Zickler D \& Kleckner N 1999 Meiotic chromosomes: integrating structure and function. Annual Review of Genetics 33 603-754.

Zhoucun A, Sizhong Z, Yuan Y, Yongxin M, Wei Z \& Li L 2006 The common variant $\mathrm{N} 372 \mathrm{H}$ in $\mathrm{BRCA} 2$ gene may be associated with idiopathic male infertility with azoospermia or severe oligozoospermia. European Journal of Obstetrics, Gynecology, and Reproductive Biology 124 61-64.

Received 28 July 2006

First decision 23 August 2006

Revised manuscript received 30 October 2006

Accepted 21 November 2006 